\title{
Study on Ablation Characteristics of Femyosecond Laser Nanoscale Processing for Aluminum Nitride and LeadZirconate Titanate Ceramics
}

C. Y.Ho ( $\nabla$ hcy2182@yahoo.com.tw)

Hwa Hsia University of Technology

Liangliang Zhou

Chizhou University

Chang-Wei Xiong

Dongguan Polytechnic

Dongkai Qiao

Guangdong University of Petrochemical Technology

\section{Research Article}

Keywords: Ultrashort pulsed Laser, Lead zirconate titanate (PZT), Aluminum nitride (AIN)

Posted Date: September 15th, 2021

DOI: https://doi.org/10.21203/rs.3.rs-903184/v1

License: (c) (i) This work is licensed under a Creative Commons Attribution 4.0 International License.

Read Full License 


\title{
Study on Ablation Characteristics of Femyosecond Laser Nanoscale Processing for Aluminum Nitride and Lead Zirconate Titanate Ceramics
}

\author{
Liangliang Zhou ${ }^{1, \dagger}$, Ching-Yen Ho ${ }^{1,2,3,4,{ }^{*}, \dagger, \ddagger}$, Chang-Wei Xiong ${ }^{3, \dagger}, \quad$ Dongkai Qiao ${ }^{4}$ \\ ${ }^{1}$ School of Mechanical and Electrical Engineering, Chizhou University, China \\ ${ }^{2}$ Department of Mechanical Engineering, Hwa Hsia University of Technology, \\ Taiwan, R.O.C \\ ${ }^{3}$ Department of Mechanical and Electrical Engineering, Dongguan Polytechnic, Dongguan \\ 523808, China. \\ ${ }^{4}$ School of Mechanical and Electrical Engineering, Guangdong University of \\ Petrochemical Technology, China \\ * Correspondence: 2298670673@qq.com; Tel.: +886-961107053 \\ $\dagger$ These authors contributed equally to this work. \\ $\ddagger$ Current address: Department of Mechanical Engineering, Hwa Hsia University of \\ Technology; hcy2182@yahoo.com.tw
}




\begin{abstract}
This paper analytically investigates an ultrashort pulsed laser nanoscale processing for aluminum nitride (AIN) and lead zirconate titanate (PZT) ceramics. Processing characteristics of an ultra-short pulsed laser is different from that of long-pulsed laser due to ultrahigh intensity, ultrahigh power, and ultrashort time. The ultrasmall processing for materials can achieved by an ultra-short pulsed laser. This study proposes a model to analyze an ultrashort pulsed laser nanoscale processing for aluminum nitride (AIN) and lead zirconate titanate (PZT) ceramics. The effects of optical penetration absorption and thermal diffusion on temperature are also discussed. The results reveal that the variation of ablation rate with laser fluences predicted by this work agrees with the available measured data for an ultrashort pulsed laser processing for AIN and PZT. For femtosecond lasers, the optical absorption and thermal diffusion, respectively, governs the ablated depth per pulse at the low and high laser fluences. The thermal diffusion length is small relative to the optical penetration depth for femtosecond laser. The optical penetration absorption governs the temperature in the workpiece. On the other hand, for the picosecond laser, the thermal diffusion length is large compared to the optical penetration depth. The thermal diffusion determines the temperature in the workpiece.
\end{abstract}

Keywords: Ultrashort pulsed Laser; Lead zirconate titanate (PZT), Aluminum nitride (AIN). 


\section{Introduction}

A femtosecond laser was employed to pattern an aluminum nitride (AIN). The AIN film was patterned precisely only little changes to the material structure of the film surface for a femtosecond laser processing [1]. AIN thin film was usually employed as a buffer layer which provides a strain between sapphire and AIN. This misfit strain makes the buffer layer to be an important structure for GaN-based devices [2] and the buffer layer is also important for the growth of epitaxial gallium nitride ( $\mathrm{GaN}$ ) layers on sapphire substrates, which promoted the development of GaN electronic and optoelectronic devices [3]. AIN thin film is also evaluated to be important substrate materials since the lattice constant and thermal expansion are approximate between the AIN and the GaN or AIGaN epitaxial layers [4]. AIN is a wide band gap semiconductor material. Its applications include radio frequency filters [5], ultraviolet (UV) solid state light sources [6], acoustic resonators [5], and photodetectors [7].

Lead zirconate titanate (PZT) thin films embedded in micro electro-mechanical systems (MEMS) can enhance efficiency and reduce size of MENS devices. PZT thin films can be formed by laser deposition MENS [8] and can work as resonators [9] and sensors [10]. The properties of PZT thin films are significantly related to the crystallization and the microstructure [11]. PZTs in MENS must be very high precision, speed and good controllability. PZTs are not easy to be machined on a micrometer scale with traditional methods due to its high hardness and brittleness. Therefore, an ultrafast laser is applicable to achieve this precision (process PZT)[12].

A nanosecond ultraviolet (UV) laser is used to pattern the electrodes on thick graphite oxide (GO) free standing films [13]. Direct laser writing (DLW) is a suitable technique for three-dimensional (3D) micro- and even nanostructuring [13]. Femtosecond laser processing for optical materials is a good technology to the production of high-quality micro- and 
nanofabrication of functional devices [14]. Femtosecond Laser Processing of Nano-Crystalline CVD Diamond Coating was studied [15]. This paper proposes an analytical model to study the femtosecond laser processing of AIN and PZT ceramics. The depth per pulse versus laser fluence of AIN and PZT ablated by a femtosecond laser is predicted and compared with the measured data. Ablation characteristics of a femtosecond laser processing for AIN and PZT is analyzed. The effects of optical penetration absorption and thermal diffusion on temperature will also be discussed.

\begin{tabular}{|llll|}
\hline Nomenclature & & \\
$c$ & volume heat capacity & $t$ & time \\
$\mathrm{D}$ & thermal diffsion length $=\sqrt{\tau_{0} k / c}$ & $T$ & temperature in the workpiece \\
$f$ & solid-vapor interface & $T_{v}$ & evaporation temperature of materials \\
$h$ & heat convection coefficient & $T_{\infty}$ & the ambient temperature \\
$\mathrm{J}$ & Laser fluence $3 Q \tau_{0} /\left(\pi \sigma^{2}\right)$ & $\mathrm{h}$ & height in the polar coordinates \\
$K$ & thermal conductivity & $\sigma$ & energy-distribution radius \\
$L$ & thickness of workpiece & $\delta$ & optical absorption length \\
$Q$ & laser power & $\tau_{0}$ & pulse duration at FWHM \\
$r$ & radius in the polar coordinates & $\gamma$ & latent heat of vaporization \\
$R$ & reflectivity & $\rho$ & mass density \\
\hline
\end{tabular}

\section{Analysis}

A model is developed for an ultrashort pulsed laser processing of PZT and AIN. Different from metals full of free electrons using two-temperature model due to the difference of temperature between electrons and lattices at the duration of laser pulse, PZT and AIN ceramics employ the thermal transport model based on phonons as carriers. Therefore, PZT and AIN The model in the polar coordinates can be written

$$
c \frac{\partial T}{\partial t}=k\left[\frac{1}{r} \frac{\partial}{\partial r}\left(r \frac{\partial T}{\partial r}\right)+\frac{1}{r^{2}} \frac{\partial^{2} T}{\partial \theta^{2}}+\frac{\partial^{2} T}{\partial z^{2}}\right]+\frac{3 Q}{\pi \delta \sigma^{2}}(1-R) e^{-\left(\frac{t}{\tau_{0}}\right)^{2}-\frac{z}{\delta}-3\left(\frac{r^{2}}{\sigma^{2}}\right)}
$$

The number 3 in Eq. (1) are taken to assure 90 percent of laser energy included within 
the energy-distribution radius. The pulse duration for a femtosecond laser is about on the order of $10^{-15}$ seconds which is shorter than relaxation time of thermal diffusion on the order of $10^{-12}$ seconds in AIN or PZT ceramics. Hence, before the relaxation time of thermal diffusion is arrived during the laser pulse, the heat conduction the $r-, \quad \theta-$, and $z$-directions is assumed to be negligible because the heat cannot diffuse in time. On the other hand, after he relaxation time of thermal diffusion is arrived, the main heat diffusion is in the z-direction because the workpiece employed in this study is about $10^{-3} \mathrm{~m}$ in the $\mathrm{z}$-direction and infinite size in the r-direction. Eq. (1) can be, therefore, written as

$$
c \frac{\partial T}{\partial t}=k \frac{\partial^{2} T}{\partial z^{2}}+\frac{3 Q}{\pi \delta \sigma^{2}}(1-R) e^{-\left(\frac{t}{\tau_{0}}\right)^{2}-\frac{z}{\delta}-3\left(\frac{r^{2}}{\sigma^{2}}\right)}
$$

Material absorption for laser is recognized to be volume absorption. When the optical penetration depth is very small, the optical surface absorption is achieved. It is assumed that the heat on the surface of workpiece is transported into the ambient by convection. The ambient temperature and vaporization temperature are, respectively, set at bottom surface of workpiece and solid-vapor interface.

$$
T(L, t)=T_{\infty},-k \frac{\partial T(0, t)}{\partial z}+h\left[T(0, t)-T_{\infty}\right]=0, T(f, t)=T_{e}
$$

The location of material removal is assumed to be at the solid-vapor interface (ablation interface). The balance of thermal energy at the solid-vapor interface is

$$
-k \frac{\partial T}{\partial z}=\rho \gamma \frac{\partial f}{\partial t}
$$

The nondimensional parameters are defined as 


$$
\theta=\frac{T-T_{\infty}}{T_{e}-T_{\infty}} ; \tau=\frac{t}{\tau_{0}} ; \xi=\frac{z}{L} ; \tau 0 L=\frac{\tau_{0}}{\frac{c L^{2}}{k}}=\frac{\tau_{0}}{\tau L}=\frac{D^{2}}{L^{2}} ; H=\frac{h L}{k} ; R=\frac{r}{L} ; \quad F=\frac{f}{L}
$$

Therefore, the nondimensional Eq. (2) can be written as

$$
\frac{\partial \theta}{\partial \tau}=\frac{D^{2}}{L^{2}} \times \frac{\partial^{2} \theta}{\partial \xi^{2}}+\frac{J}{\delta c\left(T_{e}-T_{\infty}\right)}(1-R) e^{-\tau^{2}-\frac{L \xi}{\delta}-3\left(\frac{L R}{\sigma}\right)^{2}}
$$

The symbols, D/L and $\delta / \mathrm{L}$, in Eq. (5), respectively, represents the nondimensional thermal diffusion length and optical penetration depth. When the nondimensional thermal diffusion length is small enough relative to the nondimensional optical penetration depth, the thermal diffusion term can be neglected. Therefore, the temperature in Eq. (5) is governed by the direct optical penetration absorption of incident laser pulse. On the other hand, when the nondimensional optical penetration depth is small enough relative to the nondimensional thermal diffusion length, the optical penetration absorption only occurs on the worpiece surface and the temperature inside the workpiece is not directly affected by the optical penetration absorption on the workpiece surface. For femtosecond pulsed laser, the laser pulse duration is on the order of $10^{-15} \mathrm{~s}$. However, the relaxation time of thermal diffusion, $c L^{2} / k$, is on the order of $10^{-12} \mathrm{~s}$ for materials. The ratio of ultrashort laser pulse duration to thermal diffusion time is far smaller than one. Therefore, the thermal diffusion term of the right-handed side in Eq. (5) can be neglected because the order of the other nondimensional terms is near one.

The nondimensional initial condition and boundary conditions are

$$
\theta(F, \tau)=1,-\frac{\partial \theta(0, \tau)}{\partial \xi}+H \theta(0, \tau)=0, \theta(1, \tau)=0
$$

The nondimensional relation of energy balance at solid-vapor interface is 


$$
-\frac{\partial \theta}{\partial \xi}=\Omega_{l} \frac{\partial F}{\partial \tau}
$$

where

$$
\Omega_{l}=\frac{\rho \gamma L^{2}}{k\left(T_{e}-T_{\infty}\right) \tau_{0}}
$$

The method of Laplace transform is employed to obtain the solution of Eq. (5). If the Laplace transform of the temperature $\theta$ is symbolized by $\Theta$, the Eq. (6) in the Laplace domain yields

$$
s \Theta=\tau 0 L \times \frac{d^{2} \Theta}{\partial \xi^{2}}+\frac{J}{\delta c\left(T_{e}-T_{\infty}\right)}(1-R) e^{-\frac{L \xi}{\delta}-3\left(\frac{L R}{\sigma}\right)^{2}} \frac{\sqrt{\pi}}{2} e^{\frac{s^{2}}{4}} \operatorname{erfc}\left(\frac{s}{2}\right)
$$

In the similar way, the nondimensional initial condition and boundary conditions in the Laplace domain are

$$
\Theta(\xi, 0)=0, \quad \Theta(1, s)=0,-\frac{\partial \Theta(0, s)}{\partial \xi}+H \Theta(0, s)=0 \Theta(\Gamma, s)=\frac{1}{s}
$$

The general solution of Eq. (10) with the initial condition in Laplace domain is

$$
\Theta=c 1 \times e^{-\sqrt{\frac{s}{\tau 0 L}} \zeta}+c 2 \times e^{\sqrt{\frac{s}{\tau 0 L}} \zeta}-\frac{\frac{J}{\delta c\left(T_{e}-T_{\infty}\right)}(1-R) e^{-\frac{L \xi}{\delta}-3\left(\frac{L R}{\sigma}\right)^{2}} \frac{\sqrt{\pi}}{2} e^{\frac{s^{2}}{4}} \operatorname{erfc}\left(\frac{s}{2}\right)}{\left(\tau 0 L \times \frac{L^{2}}{\delta^{2}}-s\right)}
$$

The temperature is finite for $\zeta \rightarrow \infty$ and the boundary condition on the surface of workpiece determines the constant c1. Therefore,

$$
\Theta=\Omega_{1} \frac{e^{\frac{s^{2}}{4}} \operatorname{erfc}\left(\frac{s}{2}\right)}{\left(\frac{D^{2}}{\delta^{2}}-s\right)} \times\left(\frac{H-\frac{L}{\delta}}{H-\sqrt{\frac{L^{2} S}{D^{2}}}} e^{-\sqrt{\frac{L^{2} s}{D^{2}} \xi}}-e^{-\frac{L \xi}{\delta}}\right)
$$


where

$$
\Omega_{1}=\frac{J(1-R) \sqrt{\pi} e^{-3\left(\frac{L R}{\sigma}\right)^{2}}}{2 \delta c\left(T_{d}-T_{\infty}\right)}
$$

The first term and second term in the right-handed side of Eq. (13) are, respectively, related to the effects of thermal diffusion and direct optical absorption on the temperature in the workpiece.

The nondimensional temperature in time domain is obtained by taking the inverse of Laplace transform for Eq. (13).

$$
\begin{aligned}
& \theta=\Omega_{1}\left\{\sqrt{\tau_{0 L}}\left(H-\frac{L}{\delta}\right) e^{\left(\frac{\tau_{0 L} L^{2}}{2 \delta^{2}}\right)^{2}} \int_{0}^{\tau}\left[\frac{1}{\sqrt{\pi \tau}} e^{-\frac{\xi^{2}}{4 u \tau_{0 L}}}+\left(\sqrt{\tau_{0 L}} H\right) e^{-H \xi} e^{\tau_{0 L} H^{2} u}\right] e^{\frac{\tau_{0 L} L^{2}(\tau-u)}{\delta^{2}}}\right. \\
& \left.\left[\operatorname{erf}\left(\tau-u+\frac{\tau_{0 L} L^{2}}{2 \delta^{2}}\right)-\operatorname{erf}\left(\frac{\tau_{0 L} L^{2}}{2 \delta^{2}}\right)\right] d u+e^{\left(\frac{\tau_{0 L} L^{2}}{2 \delta^{2}}\right)^{2}+\frac{\tau_{0 L} L^{2} \tau}{\delta^{2}}} e^{-\frac{L \xi}{\delta}}\left[\operatorname{erf}\left(\tau+\frac{\tau_{0 L} L^{2}}{2 \delta^{2}}\right)-\operatorname{erf}\left(\frac{\tau_{0 L} L^{2}}{2 \delta^{2}}\right)\right]\right\}
\end{aligned}
$$

Combining Eq. (15) and the energy balance equation at the solid-vapor interface, one can get the relation at the solid-vapor interface.

$$
\begin{aligned}
& \Omega_{1}\left\{\sqrt{\tau_{0 L}}\left(H-\frac{L}{\delta}\right) e^{\left(\frac{\tau_{0 L} L^{2}}{2 \delta^{2}}\right)^{2}} \int_{0}^{\tau}\left[\frac{1}{\sqrt{\pi \tau}}\left(\frac{F}{2 u \tau_{0 L}}\right) e^{-\frac{F^{2}}{4 u \tau_{0 L}}}+\left(\sqrt{\tau_{0 L}} H^{2}\right) e^{-H F} e^{\tau_{0 L} H^{2} u}\right] e^{\frac{\tau_{0 L} L^{2}(\tau-u)}{\delta^{2}}}\right. \\
& \left.\left[\operatorname{erf}\left(\tau-u+\frac{\tau_{0 L} L^{2}}{2 \delta^{2}}\right)-\operatorname{erf}\left(\frac{\tau_{0 L} L^{2}}{2 \delta^{2}}\right)\right] d u+e^{\left(\frac{\tau_{0 L} L^{2}}{2 \delta^{2}}\right)^{2}+\frac{\tau_{0 L} L^{2} \tau}{\delta^{2}}}\left(\frac{L}{\delta}\right) e^{-\frac{L F}{\delta}}\left[\operatorname{erf}\left(\tau+\frac{\tau_{0 L} L^{2}}{2 \delta^{2}}\right)-\operatorname{erf}\left(\frac{\tau_{0 L} L^{2}}{2 \delta^{2}}\right)\right]\right\}=\Omega_{2} \frac{\partial F}{\partial \tau}
\end{aligned}
$$

If the Laplace transform of the nondimensional interface $F$ is symbolized by $\Gamma$, the energy balance equation at the solid-vapor interface in Laplace domain become

$$
-\frac{\partial \Theta}{\partial \xi}=\Omega_{2} s \Gamma
$$

Substituting Eq. (13) into Eq. (17), one can get the equation at the solid-vapor interface in the nondimensional Laplace domain. 


$$
\Omega_{1} \times \frac{e^{\frac{s^{2}}{4}} \operatorname{erfc}\left(\frac{s}{2}\right)}{\left(\tau 0 L \times \frac{L^{2}}{\delta^{2}}-s\right)} \times\left(\sqrt{\frac{s}{\tau 0 L}} \times \frac{H-\frac{L}{\delta}}{H-\sqrt{\frac{s}{\tau 0 L}}} e^{-\sqrt{\frac{s}{\tau 0 L}} \Gamma}-\frac{L}{\delta} e^{-\frac{L \Gamma}{\delta}}\right)=\Omega_{2} s \Gamma
$$

The Reimann-sum approximation can transform the equation in Laplace domain into that in time domain

$$
\theta(\tau, \xi)=\frac{e^{\varepsilon \tau}}{\tau}\left\{\frac{\Theta(\varepsilon, \xi)}{2}+\operatorname{Re}\left[\sum_{n=1}^{N} \Theta\left(\varepsilon+\frac{i n \pi}{\tau}, \xi\right)(-1)^{n}\right]\right\}
$$

\section{Results and Discussion}

Figure 1 shows how the temperature in the workpiece is, respectively, influenced by optical penetration absorption and thermal diffusion for laser-pulsed duration at the order of $10^{-15} \mathrm{~s}$ (femtoseconds). The fine line and dashed line represent the temperature increase induced by optical penetration absorption and the temperature decrease induced by thermal diffusion, respectively. The temperature in AIN is symbolized the bold line which is the sum of the temperature increase induced by optical penetration absorption and the temperature decrease induced by thermal diffusion. The temperature induced by optical penetration absorption approximates the temperature in AIN except for the time longer than $7.5 \times 185$ fs (femtoseconds) because the effect of thermal diffusion is gradually obvious for long enough time. The temperature rapidly increases with time because most energy of optical penetration is used to enhance the temperature and no thermal diffusion occurs. The temperature induced by directly incident laser penetration dominate the temperature in AIN. This is because the heat cannot diffuse in time during the fs duration of laser. However, although the effect of thermal diffusion appears after the longer time, it is only slightly due to the ultrashort pulse duration of laser. The laser pulse is only one heat source which 
results in temperature increase and material ablation of AIN. On the other hand, the thermal diffusion leads to temperature decrease due to the transport of heat to low temperature. Therefore, the material ablation is first due to the penetration absorption of directly incident laser for the (femtosecond) fs laser. This case can be recognized as optical ablation. Furthermore, if the residual heat of thermal diffusion after optical ablation and can maintain material above the evaporation temperature, the thermal ablation occurs. In Eq.(5), the thermal diffusion length $D$ is related to pulse duration of laser, volume specific heat and thermal conductivity of materials. The thermal diffusion length $D$ and the ratio $D / \delta$ is small for the pulse duration at the order of femtoseconds. Therefore the thermal diffusion terms are negligible. Only the optical penetration term is left and is responsible for temperature rise. On the other hand (however), for the pulse duration at the order of picoseconds, the thermal diffusion length $D$ is large compared to the optical penetration depth $\delta$. The optical absorption is almost only on the surface of workpiece. Therefore the temperature in AIN (materials) rises only by the thermal diffusion of heat from workpiece surface.

Figure 2 shows the effects of optical penetration and thermal diffusion on the temperature in AIN for picosecond laser ablation of AIN. The fine line, and dashed line, respectively, symbolize the variations of optical penetration-induced and thermal diffusion-induced temperatures with time. The result temperature subjected to the optical-penetration and thermal-diffusion effects is denoted by the bold line. The dashed line is almost consistent with the bold line. This indicates that the thermal diffusion-induced temperature governs the temperature in AIN irradiated by picosecond laser. The optical penetration-induced temperature is very slightly greater than zero at the beginning of pulse 
duration for picosecond laser and later almost zero. The reason is because the optical penetration depth is very small relative to the thermal diffusion length for picosecond laser ablation of AIN. Therefore, the incident laser irradiating material only exchanges energy with the surface of workpiece and hardly penetrates into materials. The effect of incident laser irradiating the workpiece surface on AIN temperature can be only by means of thermal diffusion. The location of the AIN temperature recorded in Fig. 2 is $200 \mathrm{~nm}$ below the surface of workpiece. The laser heat on the surface of workpiece is first transported to the location by thermal diffusion and then the heat at the location is diffused into other places. Consequently, the AIN temperature at about $200 \mathrm{~nm}$ below the surface of workpiece initially rises to maximum with time and then gradually descends with time.

Figure 3 shows that the ablation rate of AIN varies with the logarithm of laser fluences for the ablated depth per pulse predicted by this work and measured by the published paper [16]. The triangle symbols and solid lines, respectively, denote the variation of measured and predicted ablation rate with laser fluence. The variation of ablation rate with laser fluence predicted agrees with that measured by the published paper [16]. The ablation rate increases with the logarithm of laser fluences. The increase of ablation rate with the logarithm of laser fluences seems to be specially fast for high laser fluence. The dashed line is the straight line fitting the variation of ablation rate with the logarithm of laser fluences at low laser fluences, which fllows logarithmic Beer's law (Bremsstrahlung's absorption ). The logarithmic Beer's law indicates the ablated depth per pulse is linearly proportional to the product of optical absorption length and the logarithm of the ratio of laser fluence to laser fluence threshold for optical ablation $\left(D_{\circ} \sim l_{0} \log \left(\phi / \phi_{\text {oth }}\right)\right.$ where $D_{\circ}$, lo, $\phi$, and $\phi_{\text {oth }}$, symbolize the ablation depth per pulse due to direct optical penetration absorption, optical absorption length, laser fluence, and laser fluence threshold for optical ablation. This indicates that the ablated depth of AIN by ultrafast pulse laser 
mainly depends on the optical absorption length at low laser fluences. The optical penetration absorption of AIN for ultrafast pulse laser determines the ablated depth per pulse at low laser fluences. For high laser fluences, the sufficient residual laser energy can diffuse into the ambient region and maitain the material temperature above the evaporated temperature after the optical penetration absorption of AIN for ultrafast pulse laser. In this stage, the ablation depth per pulse is linearly proportional to the product of thermal diffusion length and the logarithm of the ratio of laser fluence to laser fluence threshold for thermal ablation ( $D_{h} \sim I_{h} \log (\phi / \phi h t h)$ where $D_{h}, I_{h}, \phi$, and $\phi_{h t h}$, symbolize the ablation depth per pulse due to thermal diffusion, thermal diffusion length, laser fluence, and laser fluence threshold for thermal ablation. The laser fluence threshold of optical ablation is about $8000 \mathrm{~J} / \mathrm{m}^{2}$ and the laser fluence threshold of thermal ablation is about $200000 \mathrm{~J} / \mathrm{m}^{2}$.

The variation of the ablated depth per pulse with laser fluences is plotted in Fig. 4 for the ultrafast laser ablation of AIN. The difference between Fig. 3 and Fig. 4 is the scale of the horizontal axis. The horizontal axises in Fig. 3 and Fig. 4 are, respectively, scaled by the linearity and logarithm of laser fluences. The increase of ablation rate with laser fluences in Fig. 3 at high laser fluence is slightly more slower than that at low laser fluences. This possible reason is because the thermal ablation of the residual laser energy occurs at high laser fluences after the directly incident ultrafast laser pulse conducts optical penetration ablation due to optical absorption length. In this linear horizontal axis of laser fluences, the solid line predicted by this work agrees with the triagle symbols measured by the published paper [16] and the ablation rate still increases with the increasing laser fluences although the increase of ablation rate with laser fluences in at high laser fluence is slightly more slower than that at low laser fluences different from Fig. 3.

The relation of the ablation rate of PZT to laser fluence is plotted in Fig. 5 for the 
prediction of this work and the measurement of the published paper [17]. The solid line and triangle symbols, respectively, stand for the ablated depth per pulse versus laser fluence predicted by this work and measured by Di Maio et al [17]. The ablated depth per pulse versus laser fluence predicted by this work agrees with that measured by Di Maio et al. The ablation rate increases with the increasing laser fluence. However, the increase of the ablation rate with the laser fluence is faster at low laser fluences than that at high laser fluences. The possible reason is because the removal of materials by an ultrafast pulse laser at high laser fluences is governed by the residual heat after the optical penetration absorption of the directly incident laser energy. If the residual heat is high enough to diffuse in time and maintains the material temperature above the evaporated point, the thermal ablation occurs. The increase of thermal ablation rate at high laser fluences is generally smaller than the increase of ablation rate of optical penetration at low laser fluences. Figure 4 shows that the ablation rate predicted by this work and measured by Di Maio et al varies with the logarithm of laser fluences for an ultrafast pulse laser ablation of PZT. The predicted and measured ablation rates are linearly proportional to the logarithm of laser fluences. This also shows that the ablation rate of PZT follows the logarithmic Beer's law (Bremsstrahlung's absorption ). The removal of PZT by an ultrafast laser is due to the optical ablation of directly optical penetration absorption. In this case, the laser fluence threshold of optical ablation is about $1800 \mathrm{~J} / \mathrm{m}^{2}$. when the variation of ablation rate with the logarithm of laser fluence in Fig. 3 is compared with that in Fig. 6, Both optical and thermal ablations occur in Fig. 3 due to high laser fluences. However, only optical ablation appears in Fig. 6 due to low laser fluences.

\section{Conclusions}

The analytical study of an ultrashort pulsed laser processing for AIN and PZT is conducted in this paper. The model proposes that the material is removed at the solid-vapor 
interface. The variation of ablation rate with laser fluences predicted by this work agrees with the available measured data for an ultrashort pulsed laser processing for AIN and PZT. The ablation rate increases with the increasing laser fluences. The increase of ablation rate with laser fluences is faster at low laser fluences than that at high laser fluences. The thermal diffusion length is small relative to the optical penetration depth for the pulse duration at the order of femtoseconds. Therefore the thermal diffusion terms are negligible. Only the optical penetration term is responsible for temperature. On the other hand, for the pulse duration at the order of picoseconds, the thermal diffusion length is large enough compared to the optical penetration depth. The optical absorption is almost only on the surface of workpiece. Therefore the temperature in materials is only determined by the thermal diffusion of heat from workpiece surface absorbing directly incident laser energy.

Acknowlegements:The support by the Natural Science Foundation of Guangdong province, China (2018A0303070004), Research Item of Natural Science of Guangdong University of Petrochemical Technology (2019rc66), the Ministry of Science and Technology under grant no. MOST 108-2221-E-146-003-, Guangdong Educational Department of Scientific Research Project under grant No. 2017GKTSCX105, Dongguan Polytechnic Scientific Research Fund through grant No. 2017a04, and Dongguan Polytechnic Quality Engineering Project under No. JGZD201826 is gratefully acknowledged.

\section{References}

1. Dong F, Li R, Wu G, Liang K, Li G, Nie Y, Gan Z, Cao Q, Wang X, Zhao Q, Liu S. An investigation of aluminum nitride thin films patterned by femtosecond laser. Applied Physics Letters 2020, 116: 154101. 
2. Moatti A, and Narayan J. High-quality TiN/AIN thin film heterostructures on c-sapphire. Acta Mater 2018,145: 134-141.

3. Liu S, Peng M, Hou C, He Y, Li M, and Zheng X. PEALD-Grown Crystalline AIN Films on Si (100) with Sharp Interface and Good Uniformity. Nanoscale Research Letters 2017, 12: 279 .

4. Wang Q, Zhao Y, Huang J, Fu D, He G, Wu L. Optimization of total resolved shear stress in AIN single crystals homoepitaxially grown by physical vapor transport method. Journal of Crystal Growth 2019, 519: 14-19.

5. Dong W, Ji X, Wang W, Li T, Huang J, Zhou T, Xu K. Investigation of surface acoustic waves anisotropy on high-quality AIN/Sapphire grown by hydride vapor phase epitaxy. Materials Research Express 2019, 6: 095903.

6. Liu S, Peng M, Hou C, He Y, Li M, and Zheng X. PEALD-Grown Crystalline AIN Films on Si (100) with Sharp Interface and Good Uniformity. Nanoscale Research Letter 2017, 12: 1-6.

7. Foronda HM, Hunter DA, Pietsch M, Sulmoni L, Muhin A, Graupeter S, KneissI M. Electrical properties of (11-22) $\mathrm{Si}$ : AIGaN layers at high $\mathrm{Al}$ contents grown by metal-organic vapor phase epitaxy. Applied Physics Letters 2020, 117: 221101.

8. Schatz A, Pantel D, and Hanemann T. Pulsed laser deposition of piezoelectric lead zirconate titanate thin films maintaining a post-CMOS compatible thermal budget. Journal of Applied Physics 2017, 122: 114502.

9. Yagubizade H, Darvishi M, Chen YY, Nguyen MD, Dekkers JM, Wiegerink RJ, Elwenspoe MC, Tas NR. Pulsed-laser deposited Pb (ZrO. 52, Ti0. 48) O3-on-silicon resonators with high-stopband rejection using feed-through cancellation. Applied physics lettler 2013, 102: 063509. 
10. Zhu C, Guo D, Ye D, Jiang S, and Huang Y. Flexible PZT-Integrated, Bilateral Sensors via Transfer-Free Laser Lift-Off for Multimodal Measurements. ACS Applied Materials \& Interfaces 2020, 12: 37354-37362.

11. Rajashekhar A, Zhang HR, Srowthi B, Reaney IM, Trolier - McKinstry S. Microstructure Evolution of In Situ Pulsed - Laser Crystallized Pb (Zr0. 52Ti0. 48) O3 Thin Films. Journal of the American Ceramic Society 2016, 99: 43-50.

12. Mauclair C, Pietroy D, Di Maio Y, Baubeau E, Colombier JP, Stoian R, Pigeon F. Ultrafast laser micro-cutting of stainless steel and PZT using a modulated line of multiple foci formed by spatial beam shaping. Optics and Lasers in Engineering 2015. 67: 212-217.

13. Kumar R, Joanni E, Singh RK, da Silva, ET, Savu R, Kubota LT, Moshkalev SA. Direct laser writing of micro-supercapacitors on thick graphite oxide films and their electrochemical properties in different liquid inorganic electrolytes. Journal of colloid and interface science $2017,507: 271-278$.

14. Choi J, Schwarz C. Advances in femtosecond laser processing of optical material for device applications. Int J Appl Glass Sci 2020,11: 480-490.

15. Wei C, Ma Y, Han Y, Zhang Y, Yang L, Chen X. Study on femtosecond laser processing characteristics of nano-crystalline CVD diamond coating. Applied Sciences 2019, 9: 4273.

16. Kim SH, Sohn IB, Jeong S. Ablation characteristics of aluminum oxide and nitride ceramics during femtosecond laser micromachining. Applied Surface Science 2009, 255: $9717-9720$.

17. Di Maio Y, Colombier JP, Cazottes P, Audouard E. Ultrafast laser ablation characteristics of PZT ceramic: Analysis methods and comparison with metals. Optics 
and Lasers in Engineering 2012, 50: 1582-1591.

\section{Figures}

Figure 1. Temperature induced by optical penetration absorption and temperature drop induced by thermal diffusion for laser-pulsed duration at the order of $10^{-15} \mathrm{~s}$ (femtoseconds).

Figure 2. Temperature induced by optical penetration absorption and thermal diffusion for laser-pulsed duration at the order of $10^{-12} \mathrm{~s}$ (picoseconds).

Figure 3. Ablation rate versus laser fluence of AIN Ceramics for the solid line predicted by this work and the triangle symbols measured by the published paper [16] with the horizontal axis of the logarithm scale.

Figure 4. Ablation rate versus laser fluence of AIN Ceramics for the solid line predicted by this work and the triangle symbols measured by the published paper [16] with the horizontal axis of the linear scale.

Figure 5. Ablation rate versus laser fluence of PZT Ceramics for the solid line predicted by this work and the triangle symbols measured by the published paper [17] with the horizontal axis of the linear scale.

Figure 6. Ablation rate versus laser fluence of PZT Ceramics for the solid line predicted by this work and the triangle symbols measured by the published paper [17] with the horizontal axis of the logarithm scale 


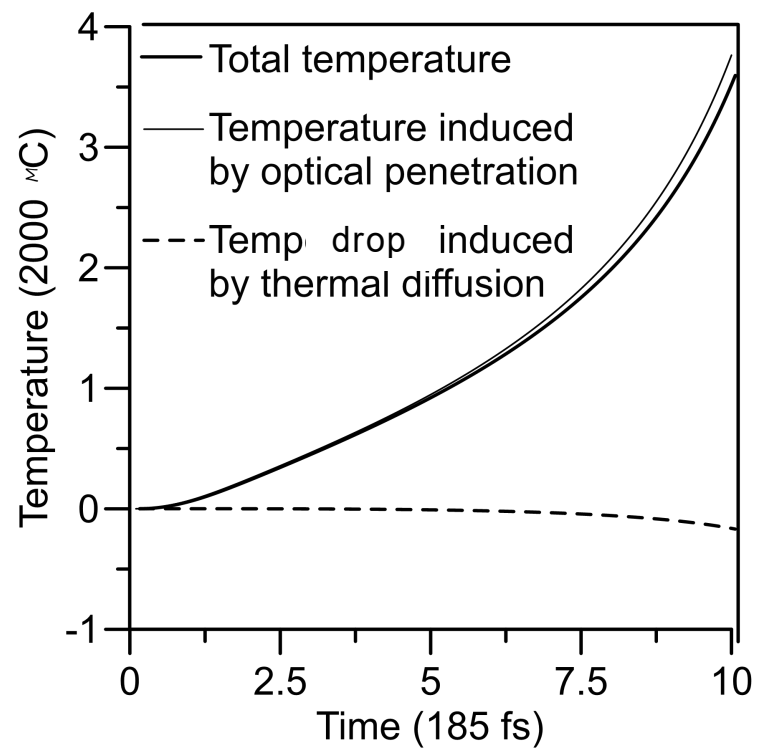

Figure 1. Temperature induced by optical penetration absorption and temperature drop induced by thermal diffusion for laser-pulsed duration at the order of $10^{-15} \mathrm{~s}$ (femtoseconds). 


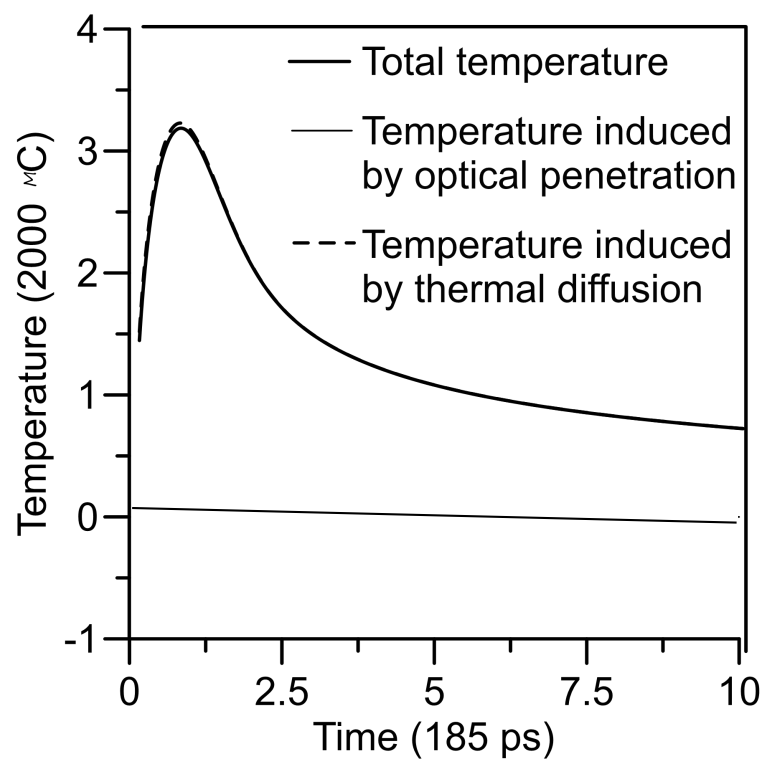

Figure 2. Temperature induced by optical penetration absorption and thermal diffusion for laser-pulsed duration at the order of $10^{-12} \mathrm{~s}$ (picoseconds). 


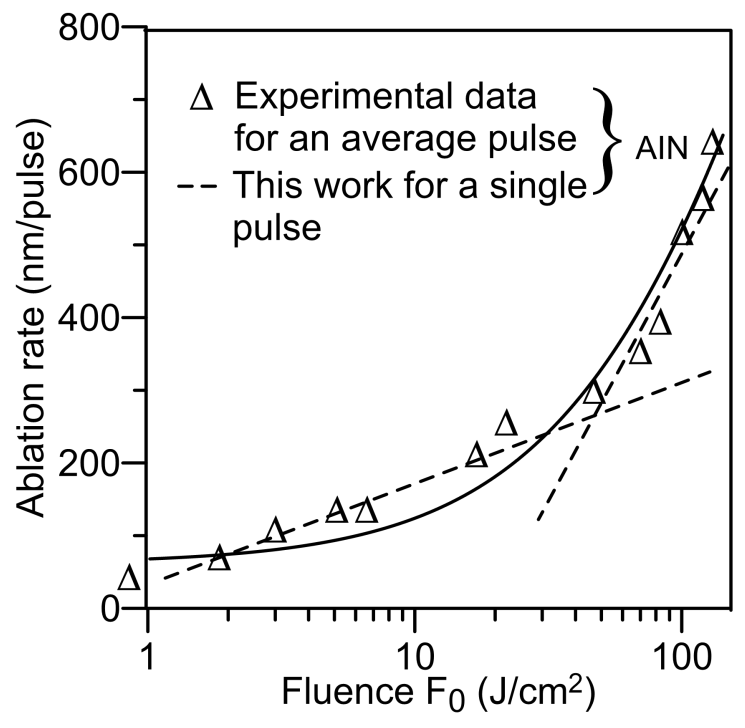

Figure 3. Ablation rate versus laser fluence of AIN Ceramics for the solid line predicted by this work and the triangle symbols measured by the published paper [16] with the horizontal axis of the logarithm scale. 


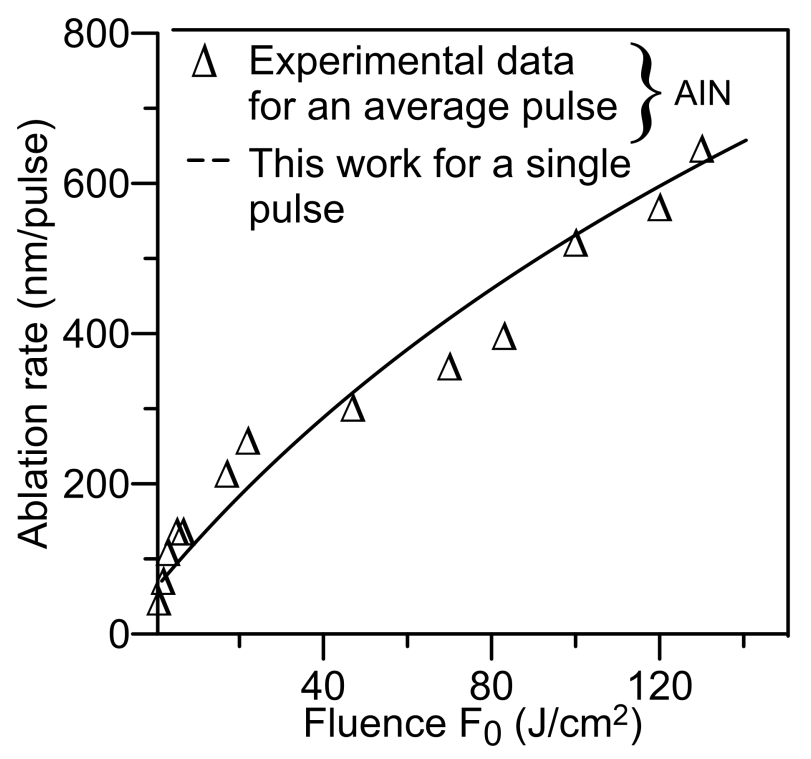

Figure 4. Ablation rate versus laser fluence of AIN Ceramics for the solid line predicted by this work and the triangle symbols measured by the published paper [16] with the horizontal axis of the linear scale. 


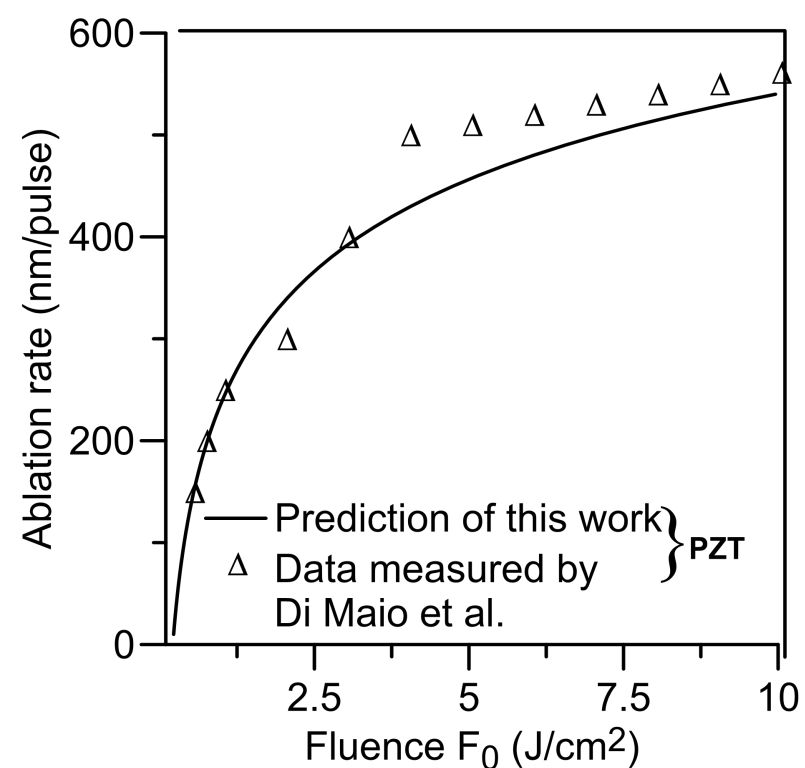

Figure 5. Ablation rate versus laser fluence of PZT Ceramics for the solid line predicted by this work and the triangle symbols measured by the published paper [17] with the horizontal axis of the linear scale. 


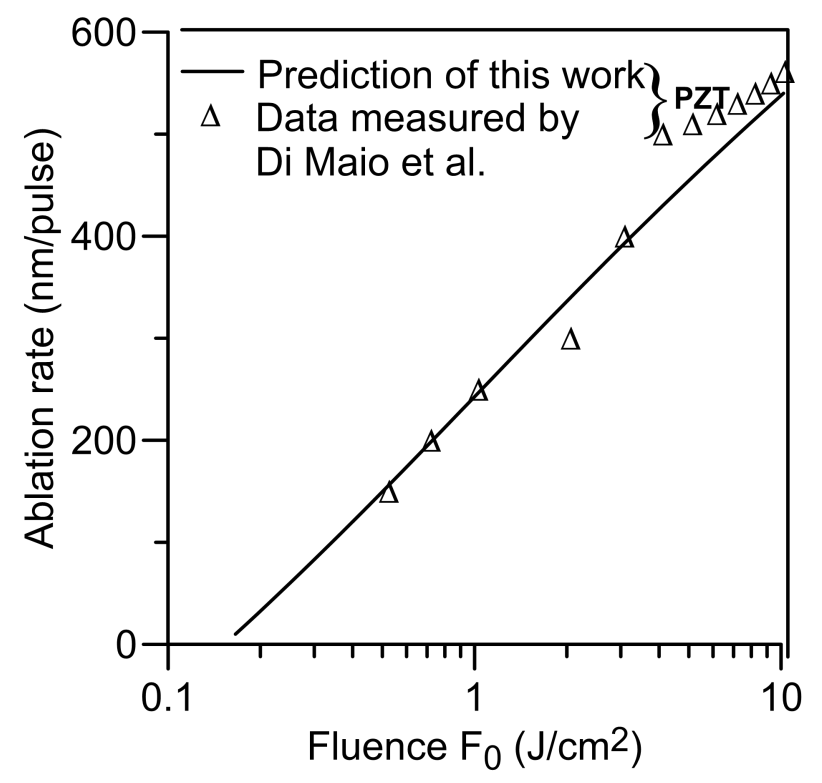

Figure 6. Ablation rate versus laser fluence of PZT Ceramics for the solid line predicted by this work and the triangle symbols measured by the published paper [17] with the horizontal axis of the logarithm scale. 